The Astrophysical Journal, 542:404-410, 2000 October 10

(c) 2000. The American Astronomical Society. All rights reserved. Printed in U.S.A.

\title{
GAS-PHASE INFRARED PHOTODISSOCIATION SPECTROSCOPY OF CATIONIC POLYAROMATIC HYDROCARBONS
}

\author{
Jos Oomens, ${ }^{1}$ ANDré J. A. van RoiJ, ${ }^{2}$ Gerard MeiJer, ${ }^{1,2}$ AND Gert von Helden ${ }^{1}$ \\ Received 2000 May 19; accepted 2000 May 26
}

\begin{abstract}
Infrared spectra of gas-phase cationic naphthalene, phenanthrene, anthracene, and pyrene are recorded in the $500-1600 \mathrm{~cm}^{-1}$ range using multiphoton dissociation in an ion trap. Gas-phase polyaromatic hydrocarbons are photoionized by an excimer laser and stored in a quadrupole ion trap. Subsequent interaction with the intense infrared radiation of a free electron laser that is tuned in resonance with an infrared-allowed transition of the ion leads to sequential multiphoton absorption facilitated by rapid intramolecular vibrational redistribution. Absorption of more than 50-100 infrared photons raises the internal energy to above the dissociation threshold, leading eventually to fragmentation of the ion. Mass selective detection of the cationic species stored in the trap yields the infrared absorption spectrum of the parent ion.
\end{abstract}

Subject headings: infrared: ISM: lines and bands - methods: laboratory - molecular data techniques: spectroscopic

\section{INTRODUCTION}

At least some of the unidentified infrared (UIR) emission bands observed from the interstellar medium (ISM) are believed to be due to polyaromatic hydrocarbons (PAHs) and, in particular, their cations (Leger \& Puget 1984; Allamandola, Tielens, \& Barker 1985, 1989; Joblin et al. 1996; Cook et al. 1996; Allamandola, Hudgins, \& Sandford 1999; Sloan et al. 1999). PAHs are the most stable hydrocarbons and are believed to constitute a substantial fraction of the interstellar carbon. Especially in those regions of the ISM where UV radiation densities are high, PAHs are believed to occur predominantly in their ionized form. Absorption of deep UV photons and rapid internal conversion raises the vibrational energy of the ion. Intramolecular vibrational redistribution (IVR) randomizes the vibrational state distribution to temperatures $T_{\text {vib }}$ comparable to $300-1000 \mathrm{~K}$ (Le Coupanec, Rouan, \& Léger 1998). Because of the extremely low collision rate, the ions undergo radiative cooling, thereby emitting infrared photons of specific wavelengths, known as the UIR bands (Allamandola et al. 1989).

Despite the astrophysical relevance of PAH infrared spectra, little laboratory gas-phase data are available for comparison because of the difficulty of creating sufficient ion densities to apply standard infrared spectroscopic techniques. To date, most laboratory information is obtained from matrix isolation spectroscopy, in which neutral PAHs and their cationic and anionic counterparts are embedded in an inert gas matrix at cryogenic temperatures (Szczepanski et al. 1992, 1993; Vala et al. 1994; Hudgins, Sandford, \& Allamandola 1994; Hudgins \& Allamandola 1995a, 1995b). Not only is it difficult to distinguish the absorption features of ionic species from those belonging to the neutrals (which outnumber the ions by far), in addition, the precise effects of the matrix on the spectrum are unknown (Joblin et al. 1994).

\footnotetext{
${ }^{1}$ FOM-Institute for Plasma Physics "Rijnhuizen," P.O. Box 1207, 3430BE Nieuwegein, The Netherlands; joso@rijnh.nl, gerardm@rijnh.nl, gertvh@rijnh.nl.

2 Department of Molecular and Laser Physics, University of Nijmegen, P.O. Box 9010,6500GL Nijmegen, The Netherlands; Andre.vanRoij@ sci.kun.nl.
}

The gas-phase infrared spectrum of the cationic naphthalene-Ar van der Waals complex has recently been recorded by Piest, von Helden, \& Meijer (1999a) via a threelaser resonance scheme for resonant two-photon ionization followed by infrared cluster dissociation (Piest et al. 1999b; Fujii et al. 2000). Since the Ar atom has particularly little effect on the vibrational energies of (poly)aromatic molecules, this so-called "messenger" technique (Okumura et al. 1990) yields the linear infrared absorption spectrum of the jet-cooled naphthalene ion.

Here we follow a different approach in order to investigate the gas-phase infrared spectrum of PAH ions. Using a free electron laser (FEL), ionized PAHs are multiphotondissociated while being confined in an ion trap, and the fragments are mass-selectively detected. In contrast to techniques based on the dissociation of weakly bound clusters as mentioned above, in the present study bare PAH cations are fragmented, requiring the absorption of many $(>50)$ infrared photons. The sequential multiphoton absorption process relies strongly on rapid IVR, which is known to occur on (sub)nanosecond timescales in these large molecules (Felker \& Zewail 1985). Scanning the FEL wavelength while monitoring the fragmentation yield gives the infrared spectrum of the ion.

Spectra were recorded at (or slightly above) room temperature, which appears to be in contrast with the conditions present in the ISM. However, in order for a molecule to emit in the mid-infrared range, its vibrational temperature should be about 300-1000 K (Le Coupanec et al. 1998). In the ISM, this energy is provided by rapid vibronic relaxation after the absorption of a UV photon. The vibrational temperature has distinct effects on both the position and the line width of infrared transitions (Brenner \& Barker 1992; Schlemmer et al. 1994); e.g., Joblin et al. (1995) found that the vibrational band centers of neutral PAHs shift to longer wavelengths by typically a few $\mathrm{cm}^{-1}$ per $100 \mathrm{~K}$.

Since species in the ISM are exposed to energetic photons, the photophysics of PAH ions are of great interest. Photodissociation rates of molecular or ionic species scale with their total internal energy, and different dissociation channels likely possess different dependencies (see, e.g., Gotkis et al. 1993 for naphthalene and phenanthrene). IVR 
rates of highly excited PAHs (Felker \& Zewail 1985) are generally many orders of magnitude higher than dissociation rates so that dissociation originates from a thermal bath of vibrational states. Photofragmentation patterns of a great variety of PAH cations under irradiation by a Xe lamp are reported by Ekern et al. (1998). In view of the UIR hypothesis, it is especially interesting to compare photodissociation rates with infrared radiative lifetimes as a function of the internal energy of the ion (Jochims, Baumgärtel, \& Leach 1999). Boissel (1995) studied photofragmentation of cationic anthracene and pyrene and described the process in terms of a competition between radiative cooling and absorption of UV-visible photons. Compared with the broadband light sources used in some of these studies, the high spectral brightness of the FEL applied here is able to induce different fragmentation patterns.

\section{EXPERIMENTAL}

A quadrupole ion trap (R. M. Jordan Co.), made accessible to the laser beams, and a $20 \mathrm{~cm}$ long Wiley-McLaren type time of flight (TOF) mass spectrometer, equipped with a multichannel plate (MCP) detector, form the heart of the experimental apparatus shown in Figure 1. Vapor phase molecules that effuse toward the center of the trap are ionized by the focused radiation of an ArF excimer laser (Lambda Physik EMG 100). Low vapor pressure species can be heated in a quartz oven. A shutter in front of the
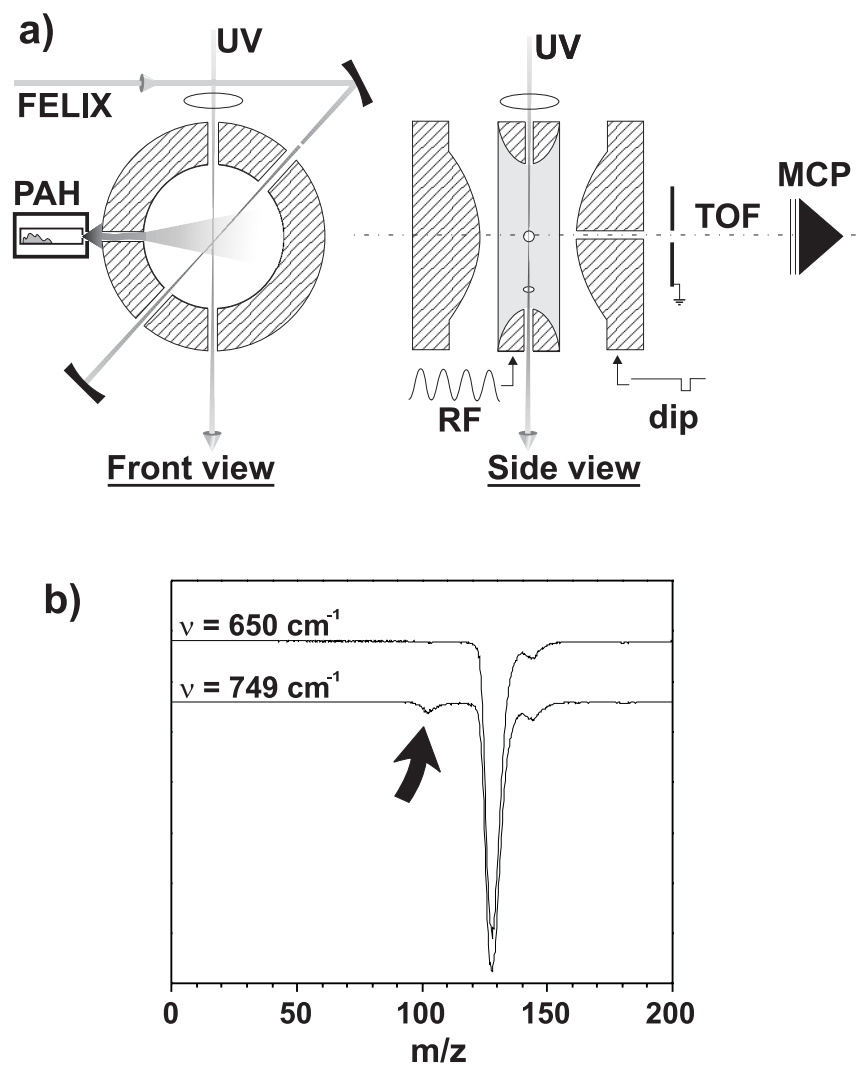

FIG. 1.-(a) Front and side cuts through the ion trap used in the experiments. The ring electrode is fed with a $1 \mathrm{MHz} \mathrm{RF}$ voltage while one of the endcaps is used as pulsed extractor plate. The PAH sample is placed in a quartz tube that can be heated to increase the vapor pressure. (b) Time-offlight traces for naphthalene recorded on and off the resonance near 750 $\mathrm{cm}^{-1}$. The arrow indicates the appearance of the $\mathrm{C}_{8} \mathrm{H}_{6}^{+}$fragment ions when FELIX is tuned in resonance. oven, which opens for about $5 \mathrm{~ms}$ just before the ionization laser pulse, reduces the deposition load on the trap and on other parts inside the vacuum chamber. The quadrupole ion trap (Paul 1990; Todd \& Penman 1991) consists of a $2 \mathrm{~cm}$ inner diameter toroidal ring electrode and two hyperbolically shaped endcap electrodes. The three electrodes are biased with typically $900 \mathrm{~V}$, and extraction of the trapped ions toward the detector is achieved by applying a voltage $\operatorname{dip}(-200 \mathrm{~V})$ on the endcap nearest to the detector. In addition to the bias voltage, the ring electrode is fed with a 1 $\mathrm{MHz}$ radio frequency (RF) voltage with an amplitude depending on the mass of the ionic species investigated (typically 1000-2000 V p-p). For about $2 \mathrm{~ms}$ after the ArF laser pulse, the RF amplitude is raised such that masses lower than that of the parent ion under study are unstable in the trap. Thus, ionic fragments formed by the intense ArF radiation are ejected from the trap, and subsequent infrared ion fragmentation is measured against zero background.

The trapped ions are irradiated with infrared laser pulses generated by the Free Electron Laser for Infrared Experiments (FELIX), which are focused in the center of the trap by a concave mirror $(R=7.5 \mathrm{~cm})$ and back-reflected in order to increase the laser fluence. FELIX (Oepts, Van der Meer, \& Van Amersfoort 1995) is continuously tunable from 5 to $250 \mu \mathrm{m}$ and produces $5 \mu \mathrm{s}$ long macropulses of up to $100 \mathrm{~mJ}$ at a $10 \mathrm{~Hz}$ repetition rate. Each macropulse consists of a train of micropulses of adjustable length (0.3-5 ps) separated by $1 \mathrm{~ns}$. The bandwidth is Fourier transformlimited and amounts to about $0.8 \%$ of the central wavelength in the present experiments. Pulse energies of about $20 \mathrm{~mJ}$ are used, which corresponds to a spectrally resolved photon flux of around $10^{23} \mathrm{~s}^{-1} \mathrm{~cm}^{-2} \mathrm{~nm}^{-1}$ averaged over a macropulse. These fluxes are about 6 orders of magnitude higher than those typically obtained using a Xe lamp (Ekern et al. 1998).

When FELIX is tuned in resonance with an allowed infrared transition, the ion can absorb several photons provided that internal relaxation occurs rapidly and/or vibrational anharmonicity is small compared with the laser bandwidth. If the internal energy has risen sufficiently, the ion will dissociate and fragment ions are formed and collected in the trap. Fragment and parent ions remain confined in the trap as long as the RF voltage is on. The trapped ions are exposed to 2 or 3 FELIX pulses before the ions are extracted from the trap and mass-analyzed with the TOF spectrometer. To empty the trap and analyze its contents, the RF voltage is switched off by the power supply ( $R$. M. Jordan Co., D-1203) and one of the endcaps is used as a (pulsed) extractor plate; an additional plate at ground potential accelerates the ions toward the MCP. The MCP transients are digitized and averaged $(20 \times)$ by a 10 bit digital oscilloscope (LeCroy 9430) and stored into a PC using LabView software.

Figure $1 b$ shows typical mass spectra for the naphthalene cation recorded on and off the resonance near $750 \mathrm{~cm}^{-1}$. Clearly, the limited resolution of our TOF instrument $(\mathrm{m} /$ $\Delta m \approx 50$ ) does not allow us to resolve the $\mathrm{H}_{2}$-loss channel, which is, for most PAHs at the energies under consideration, the major fragmentation channel (Ekern et al. 1998; Gotkis et al. 1993; Jochims et al. 1999), and spectra are therefore recorded at the $\mathrm{C}_{2} \mathrm{H}_{n}$-loss channel. Monitoring the relative fragmentation yield as function of FELIX wavelength, one obtains the infrared spectrum of the naphthalene cation as displayed in Figure 2a. The spectrum of this 


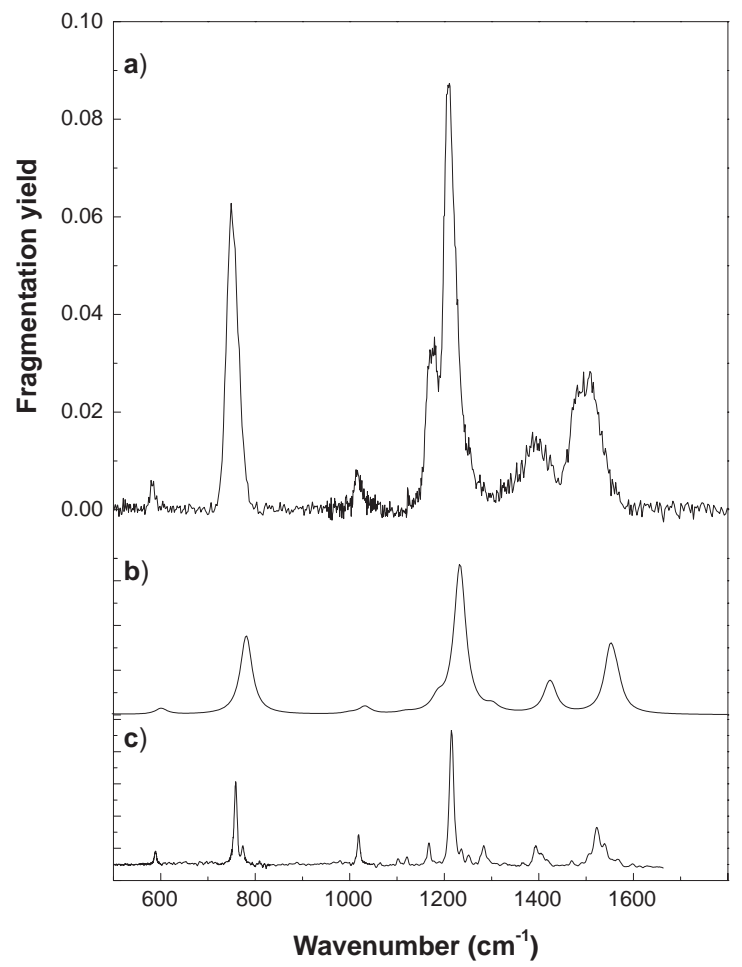

FIG. 2.-(a) Gas-phase infrared spectrum of the naphthalene cation obtained via multiphoton IR dissociation in an ion trap, compared with $(b)$ the calculated spectrum (Piest et al. 1999a) convoluted with a $30 \mathrm{~cm}^{-1}$ Lorentzian line shape and $(c)$ the jet-cooled linear absorption spectrum of the cationic naphthalene-Ar van der Waals complex of Piest et al. (1999a).

particular ion allows us to validate our technique by comparing it with known matrix, calculated, and gas-phase data (Szczepanski et al. 1992; Hudgins et al. 1994; Pauzat et al. 1992; Piest et al. 1999a).

\section{EVALUATION OF THE NAPHTHALENE CATION SPECTRUM}

Comparing our spectrum with that of Piest et al. (1999a), which has been included in Figure 2, it is evident that the two spectra are very similar. Peak positions and relative intensities compare well, as is seen in Table 1. The line width of the peaks is, however, significantly larger in this work. Not only is the ro-vibrational temperature in our experiment much higher than it is in the molecular beam of Piest et al., but multiphoton absorption processes and the occurrence of vibrational hot bands increase the line width because of anharmonic interactions (Brenner \& Barker 1992; Schlemmer et al. 1994; Joblin et al. 1995). Close inspection of the two spectra reveals that most lines in our spectrum are slightly redshifted relative to their counterparts in the linear absorption spectrum (Piest et al. 1999a), which is most likely due to vibrational (cross)anharmonicities as well (Joblin et al. 1995). In general, line positions in the two spectra coincide within 10 $\mathrm{cm}^{-1}$, except for the feature near $1500 \mathrm{~cm}^{-1}$, which, however, appears to be due to several unresolved bands in both spectra.

The spectral line width on the two strongest peaks in Figure $2 a$ is about $30 \mathrm{~cm}^{-1}$ (FWHM), which is substantially larger than the instrumental bandwidth of FELIX $(\approx 0.8 \%)$. Based on the approximate rotational constants, which are roughly the same for naphthalene and its cation (Pauzat et al. 1992), and the temperature (298 K), the FWHM width of the rotational contour is calculated to be about $16 \mathrm{~cm}^{-1}$. Convolution of this band shape with the instrumental line width yields a feature of typically $\leq 20 \mathrm{~cm}^{-1}$ FWHM, relatively independent of the band type ( $a-, b-$, or $c$-type band in an asymmetric rigid rotor). Assuming that the additional broadening is due to anharmonicities, which tend to redshift the absorption feature, a typical redshift of $5-10 \mathrm{~cm}^{-1}$ is estimated, in good agreement with the jet-cooled absorption spectrum (Piest et al. 1999a). Note that similar line widths were found for neutral gas-phase para-amino benzoic acid at room temperature in the infrared-vacuum ultraviolet (IR-VUV) double resonance experiment (Putter, von Helden, \& Meijer 1996). Furthermore, it is interesting to note that typical UIR line widths are in the $20-40 \mathrm{~cm}^{-1}$ range and are believed to be due to a combination of anharmonic shifts in vibrationally hot molecules, rapid IVR, and rotational contours at a temperature of about $100 \mathrm{~K}$ (Le Coupanec et al. 1998; Joblin et al. 1995; Rouan et al. 1992).

\section{CATION SPECTRA OF PHENANTHRENE, ANTHRACENE, AND PYRENE}

The upper panel of Figure 3 shows the spectra observed for cationic phenanthrene $\left(\mathrm{C}_{14} \mathrm{H}_{10}^{+}\right)$. Some typical features

TABLE 1

INFRARED AbSORPTION FREQUENCIES OF THE NAPHTHALENE CATION

\begin{tabular}{lcccccccc}
\hline \hline \multicolumn{2}{c}{ CALCUlATEd $^{\text {a }}$} & \multicolumn{2}{c}{$\mathrm{Na}^{+}-\mathrm{Ar}^{\mathrm{a}}$} & \multicolumn{2}{c}{ MATRIX $^{\mathrm{b}}$} & \multicolumn{2}{c}{ THIS WoRK } \\
\hline \multicolumn{1}{c}{$v_{\text {vib }}$} & \multicolumn{1}{c}{$I_{\text {rel }}$} & \multicolumn{1}{c}{$v_{\text {vib }}$} & \multicolumn{1}{c}{$I_{\text {rel }}$} & $v_{\text {vib }}$ & $I_{\text {rel }}$ & $v_{\text {vib }}$ & $I_{\text {rel }}$ \\
\hline 601 & .04 & 589 & .04 & $\ldots$ & $\ldots$ & 582 & .07 \\
781 & .53 & 759 & .53 & 759 & .27 & 749 & .76 \\
1032 & .05 & 1019 & .05 & 1023 & .05 & 1017 & .09 \\
1118 & .01 & 1121 & .01 & $\ldots$ & $\ldots$ & $\ldots$ & $\ldots$ \\
1186 & .08 & 1168 & .08 & $\ldots$ & $\ldots$ & 1173 & .20 \\
$\ldots$ & $\ldots$ & $\ldots$ & $\ldots$ & 1215 & .20 & $\ldots$ & $\ldots$ \\
1233 & 1.00 & 1215 & 1.00 & 1218 & 1.00 & 1208 & 1.00 \\
1302 & .04 & 1284 & .04 & $\ldots$ & $\ldots$ & $\ldots$ & $\ldots$ \\
1421 & .14 & 1393 & .23 & 1401 & .04 & 1394 & .17 \\
1551 & .40 & 1523 & .40 & 1519 & .10 & 1485 & .22 \\
1565 & .14 & 1539 & .14 & 1526 & .29 & 1511 & .26 \\
\hline
\end{tabular}

${ }^{\text {a }}$ Piest et al. 1999a.

${ }^{\text {b }}$ Hudgins et al. 1994. 


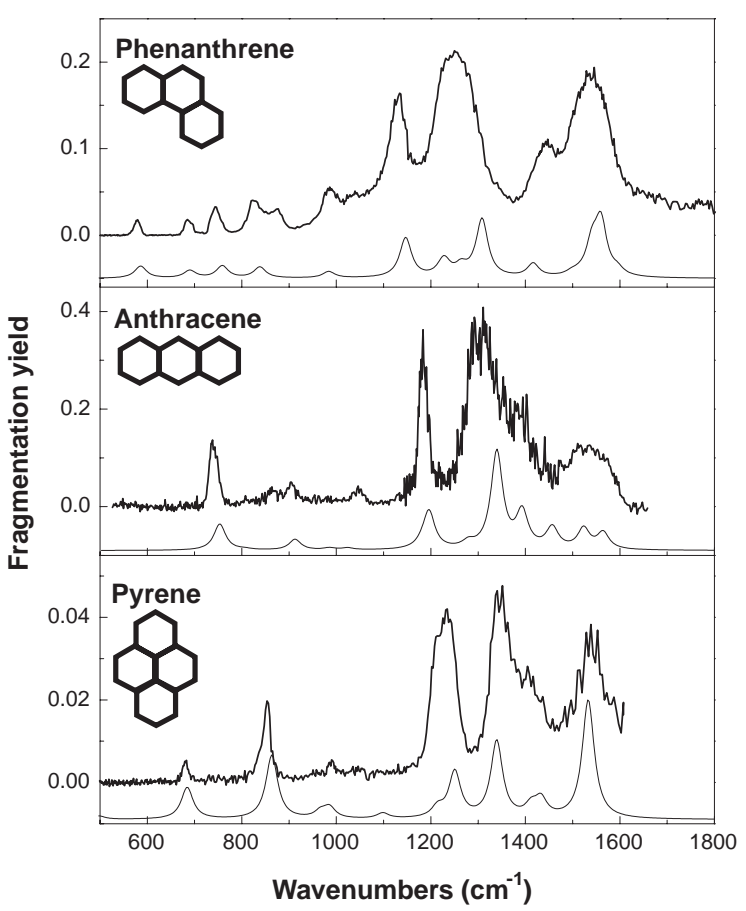

FIG. 3.-Infrared spectra of cationic phenanthrene, anthracene, and pyrene recorded via dissociation in the ion trap. Thin lines indicate calculated spectra of Langhoff (1996) convoluted with a $30 \mathrm{~cm}^{-1}$ Lorentzian line shape function.

in these spectra compare quite well with spectra obtained in matrices, as is seen in Table 2 (Hudgins \& Allamandola 1995a). In agreement with the matrix spectra, more intensity is observed toward the blue end of the spectrum, in contrast with the spectrum of neutral phenanthrene. The frequencies of the out-of-plane $\mathrm{CH}$ bending vibrations are roughly determined by the number of adjacent $\mathrm{CH}$ units on the aromatic rings of the species (Hudgins \& Allamandola

TABLE 2

Infrared Absorption Frequencies of the Phenanthrene CATION

\begin{tabular}{|c|c|c|c|c|c|}
\hline \multicolumn{2}{|c|}{ CAlculated $^{a}$} & \multicolumn{2}{|c|}{ MATRIX $^{b}$} & \multicolumn{2}{|c|}{ THIS WORK } \\
\hline$v_{\mathrm{vib}}$ & $I_{\mathrm{rel}}$ & $v_{\mathrm{vib}}$ & $I_{\text {rel }}$ & $v_{\text {vib }}$ & $I_{\text {rel }}$ \\
\hline 585.7 & .21 & 582.0 & .22 & 578 & .09 \\
\hline 689.9 & .13 & 694.5 & .09 & 684 & .08 \\
\hline 758.6 & .21 & 756.2 & .07 & 744 & .17 \\
\hline 838.1 & .19 & 836.0 & .06 & 826 & .18 \\
\hline$\ldots$ & $\ldots$ & $\ldots$ & $\ldots$ & 838 & .05 \\
\hline$\ldots$ & $\ldots$ & $\ldots$ & $\ldots$ & 874 & .13 \\
\hline 983.8 & .11 & $\ldots$ & $\ldots$ & 985 & .28 \\
\hline$\ldots$ & $\ldots$ & $\ldots$ & $\ldots$ & 1034 & .22 \\
\hline 1146.7 & .68 & $\ldots$ & $\ldots$ & 1129 & .87 \\
\hline 1221.7 & .06 & $\ldots$ & $\ldots$ & $\ldots$ & $\ldots$ \\
\hline 1228.7 & .26 & $\ldots$ & $\ldots$ & 1228 & .85 \\
\hline$\ldots$ & $\ldots$ & 1258.7 & .04 & $\ldots$ & $\ldots$ \\
\hline 1263.8 & .17 & 1267.0 & .14 & $\ldots$ & $\ldots$ \\
\hline 1308.3 & 1.00 & $\approx 1280$ & .84 & 1259 & 1.00 \\
\hline$\ldots$ & $\ldots$ & 1299.0 & .09 & $\ldots$ & $\ldots$ \\
\hline 1416.4 & .23 & $\ldots$ & $\ldots$ & 1443 & .56 \\
\hline 1540.7 & .52 & $\ldots$ & $\ldots$ & $\ldots$ & $\ldots$ \\
\hline 1559.8 & .93 & 1565 & 1.00 & 1540 & .98 \\
\hline
\end{tabular}

a Langhoff 1996.

${ }^{\text {b }}$ Hudgins \& Allamandola 1995a.
1999); e.g., phenanthrene possesses two aromatic rings with four adjacent $\mathrm{CH}$ units and one ring with doubly adjacent $\mathrm{CH}$ units. In our spectrum, these modes are observed at 744 and $826 \mathrm{~cm}^{-1}$, in rather good agreement with their matrixisolated counterparts at 756 and $836 \mathrm{~cm}^{-1}$, respectively (Hudgins \& Allamandola 1995a). The agreement for the low-frequency skeletal bending modes is even better. However, toward the blue end of the spectrum where $\mathrm{CH}$ in-plane bending and $\mathrm{CC}$ stretching modes are located, larger discrepancies are present. The four bands at 874, 985, 1034 , and $1129 \mathrm{~cm}^{-1}$ of medium to very strong intensity appear to have escaped observation in the spectrum of Hudgins \& Allamandola (1995a), although two of these bands occur in the calculations by Langhoff (1996). The strong and not fully resolved feature observed near 1247 $\mathrm{cm}^{-1}$, which appears to consist of two bands at 1228 and $1259 \mathrm{~cm}^{-1}$ in spectra taken at lower laser fluences (not shown), is redshifted by $1 \%-2 \%$ in comparison with this feature observed in the matrix $\left(\approx 1280 \mathrm{~cm}^{-1}\right)$ (Hudgins \& Allamandola 1995a) and also with the two bands calculated at 1228.7 and $1308.3 \mathrm{~cm}^{-1}$ (Langhoff 1996). A detailed study of the phenanthrene cation including the linear absorption spectrum with higher resolution will be discussed in a future publication by Piest et al. (2000).

The anthracene cation $\left(\mathrm{C}_{14} \mathrm{H}_{10}^{+}\right)$was found to fragment very efficiently. At the strong absorption features due to the in-plane $\mathrm{CH}$ bending modes, saturation of the fragmented fraction occurred, caused by severe depletion of the parent ion. The spectrum is therefore recorded with the laser power attenuated by $8 \mathrm{~dB}$, and relative intensities are rescaled accordingly, as shown in the middle panel of Figure 3. In addition, the strongest feature in the spectrum is observed slightly redshifted in the strongly saturated spectrum (1292 $\mathrm{cm}^{-1}$ ) with respect to its position in the spectrum recorded at reduced power. Despite this correction, the feature remains redshifted by about $2 \%$ with respect to the feature in the matrix isolation spectrum (Hudgins \& Allamandola 1995b) and in the calculated spectrum (Langhoff 1996), whereas the $\mathrm{CH}$ in-plane bending mode at $1186 \mathrm{~cm}^{-1}$ is observed without appreciable shift (see Table 3). The intensity ratio of the two in-plane $\mathrm{CH}$ bending modes appears to be in agreement with matrix observations (Hudgins \& Allamandola 1995b), whereas the calculations seem to underestimate the intensity of the peak at $1186 \mathrm{~cm}^{-1}$ (Langhoff 1996). The frequencies of the out-of-plane $\mathrm{CH}$ bending vibrations are established at $739 \mathrm{~cm}^{-1}$ (four adjacent $\mathrm{CH}$ units) and $905 \mathrm{~cm}^{-1}$ (nonadjacent $\mathrm{CH}$ units), corresponding to a $1 \%-2 \%$ redshift with respect to calculated (Langhoff 1996) and matrix-isolated spectra (Hudgins \& Allamandola 1995b; Szczepanski et al. 1993). Interestingly, the line at 905 $\mathrm{cm}^{-1}$ is observed slightly beyond the limit of what is normally observed for nonadjacent $\mathrm{CH}$ units, and moreover, the line is accompanied by a second feature at $862 \mathrm{~cm}^{-1}$, which was reported neither in the calculations nor in the isolation spectra. Possibly, this feature arises because of a combination mode in interaction with the $905 \mathrm{~cm}^{-1}$ fundamental, thus borrowing intensity and pushing the latter toward higher frequencies. Combination of two lowfrequency skeletal bending modes may well provide a band of the right energy and symmetry (Szczepanski et al. 1993). The absorption observed at $1047 \mathrm{~cm}^{-1}$ may correspond to the line observed by Szczepanski et al. (1993) at $1034 \mathrm{~cm}^{-1}$ and calculated at $1023 \mathrm{~cm}^{-1}$, though a blueshift of $1 \%-2 \%$ must be assumed. Toward the high-frequency end of the 
TABLE 3

INFRARED ABSORPTION FREQUENCIES OF THE ANTHRACENE CATION

\begin{tabular}{lccccc}
\hline \hline \multicolumn{2}{c}{ Calculated $^{\mathrm{a}}$} & \multicolumn{2}{c}{ Matrix $^{\mathrm{b}}$} & \multicolumn{2}{c}{ This work } \\
\hline$v_{\text {vib }}$ & $I_{\text {rel }}$ & $v_{\text {vib }}$ & $I_{\text {rel }}$ & $v_{\text {vib }}$ & $I_{\text {rel }}$ \\
\hline 753 & .27 & 748 & .26 & 739 & .38 \\
806 & .01 & $\ldots$ & $\ldots$ & 862 & .09 \\
913 & .11 & 912 & .09 & 905 & .10 \\
985 & .02 & $\ldots$ & $\ldots$ & $\ldots$ & $\ldots$ \\
1023 & .02 & $\ldots$ & $\ldots$ & 1047 & .10 \\
1182 & .03 & 1183 & .01 & $\ldots$ & $\ldots$ \\
1196 & .39 & 1189 & .70 & 1186 & .95 \\
1279 & .06 & 1290 & .06 & $\ldots$ & $\ldots$ \\
$\ldots$ & $\ldots$ & 1315 & .06 & $\ldots$ & $\ldots$ \\
1340 & 1.00 & 1341 & 1.00 & 1302 & 1.00 \\
$\ldots$ & $\ldots$ & 1353 & .31 & 1342 & .45 \\
$\ldots$ & $\ldots$ & 1364 & .04 & $\ldots$ & $\ldots$ \\
$\ldots$ & $\ldots$ & 1410 & .10 & $\ldots$ & $\ldots$ \\
1393 & .37 & 1418 & .86 & 1391 & .50 \\
1455 & .06 & 1430 & .01 & $\ldots$ & $\ldots$ \\
1459 & .16 & 1457 & .07 & $\ldots$ & $\ldots$ \\
1523 & .21 & 1540 & .15 & 1518 & .27 \\
1565 & .17 & 1586 & .14 & 1554 & .25 \\
\hline
\end{tabular}

${ }^{\text {a }}$ Langhoff 1996.

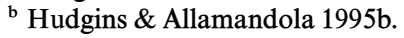

spectrum, features are poorly resolved although the most intense lines can still be discerned. For the lines at 1391, 1518 , and $1554 \mathrm{~cm}^{-1}$, the agreement with the calculations (Langhoff 1996) appears to be slightly better than with matrix frequencies, which tend to be blueshifted by about $2 \%$.

The pyrene cation $\left(\mathrm{C}_{16} \mathrm{H}_{10}^{+}\right)$spectrum is shown in the lower panel of Figure 3 in conjunction with the calculated spectrum (Langhoff 1996). Fragmentation of pyrene whereby a $\mathrm{C}_{2} \mathrm{H}_{2}$ unit is detached is uncommon (Ekern et al. 1998), and thus the signal is rather weak (compare vertical scale with that of, e.g., anthracene). From Table 4 one notices that most prominent features are observed with a redshift of typically $1.0 \%-1.5 \%$, which is likely due to the effects of vibrational anharmonicities in the present data. Of the $\mathrm{CH}$ out-of-plane bending modes, only the one due to

TABLE 4

Infrared Absorption Frequencies of the Pyrene Cation

\begin{tabular}{cccccc}
\hline \hline \multicolumn{2}{c}{ CALCULATED $^{\mathrm{a}}$} & \multicolumn{2}{c}{ MATRIX $^{\mathrm{b}}$} & \multicolumn{2}{c}{ THIS WORK } \\
\hline \multicolumn{1}{c}{$v_{\text {vib }}$} & $I_{\text {rel }}$ & \multicolumn{1}{c}{$v_{\text {vib }}$} & $I_{\text {rel }}$ & $v_{\text {vib }}$ & $I_{\text {rel }}$ \\
\hline 683.1 & .05 & $\ldots$ & $\ldots$ & $\ldots$ & $\ldots$ \\
684.7 & .24 & 690.1 & .23 & 682 & .12 \\
863.4 & .58 & 861.0 & .27 & 854 & .45 \\
966.1 & .06 & 976.5 & .09 & $\ldots$ & $\ldots$ \\
985.4 & .10 & $\ldots$. & $\ldots$ & 989 & .10 \\
1098.3 & .05 & 1102.0 & .08 & $\ldots$ & $\ldots$ \\
1214.2 & .09 & 1216.0 & .12 & 1212 & .50 \\
1250.6 & .42 & 1245.1 & .34 & 1235 & .80 \\
1339.3 & .70 & $\approx 1357$ & 1.00 & 1345 & 1.00 \\
$\ldots$ & $\ldots$ & 1361.8 & .16 & $\ldots$ & $\ldots$ \\
1412.2 & .10 & 1421.1 & .16 & 1405 & .52 \\
1433.0 & .16 & 1440.3 & .10 & $\ldots$ & $\ldots$ \\
1532.6 & 1.00 & $\approx 1553$ & .89 & 1538 & .80 \\
\hline
\end{tabular}

a Langhoff 1996.

${ }^{\mathrm{b}}$ Hudgins \& Allamandola 1995a. doubly adjacent $\mathrm{CH}$ units is observed at $854 \mathrm{~cm}^{-1}$. Our spectrum confirms that the band due to the two rings with triply adjacent $\mathrm{CH}$ units, expected to be located between 750 and $810 \mathrm{~cm}^{-1}$ (Hudgins \& Allamandola 1999), is apparently too weak to be observed, as was previously suggested by Hudgins \& Allamandola (1995a). Of the $\mathrm{CH}$ in-plane bending modes, the band calculated and observed in the matrix spectrum near $1102 \mathrm{~cm}^{-1}$ (Hudgins \& Allamandola 1995a; Vala et al. 1994) is absent in our spectrum, whereas the somewhat weaker band near $989 \mathrm{~cm}^{-1}$ is detected with a small blueshift. In agreement with the matrix spectrum, most intense bands are found in the in-plane $\mathrm{CH}$ bending and CC stretching ranges, though, toward the blue $(>1400$ $\mathrm{cm}^{-1}$ ), intensities may be underestimated because of the fading power of FELIX and the fact that the dependence on laser fluence appears to be substantially higher than linear for this ion.

\section{EXCITATION MECHANISM}

From the ionization potential of naphthalene $(8.12 \mathrm{eV})$ and the appearance energy of the $\mathrm{C}_{8} \mathrm{H}_{6}^{+}$fragment $(14.4 \mathrm{eV})$ (Gotkis et al. 1993), the energy required in order to dissociate the ion can be estimated to be $6.3 \mathrm{eV}$, corresponding to about 50 photons at $10 \mu \mathrm{m}$. In view of the line width of the infrared radiation and typical anharmonicities in vibrational potentials, true multiphoton absorption-i.e., climbing the vibrational ladder of the excited mode - is clearly an unrealistic mechanism here. A more likely process involves sequential photon absorption. Such a mechanism has been described in detail for fullerenes (von Helden et al. 1999) and is able to explain the absorption of several hundred photons per molecule, leading to thermal ionization of the fullerene (von Helden et al. 1997).

To obtain insight into the excitation mechanism, the density of vibrational states $\rho(E)$ of the naphthalene cation was evaluated using the Beyer-Swineheart algorithm (Stein \& Rabinovitch 1973) and calculated fundamental frequencies (Pauzat et al. 1992) (see Fig. 4a). Assuming that upon ionization the excess energy is mostly converted into kinetic energy, the vibrational state distribution of the ion is obtained by convoluting $\rho(E)$ with the Boltzmann factor $e^{-E / k T}$ at room temperature. Normalizing the vibrational state distribution (Fig. $4 b$ ) and multiplying with the mode energy yields the average energy per vibrational mode, which gives an integrated average energy per molecule of $1006 \mathrm{~cm}^{-1}$. Note that at room temperature, only $9 \%$ of the molecules are in the vibrational ground state (Fig. 4b), which emphasizes the influence of vibrational anharmonicity on spectral line positions. After absorption of the first photon, an ion possesses on average $2000 \mathrm{~cm}^{-1}$ of internal energy and finds itself in a region where $\rho$ amounts to around 20 states per $\mathrm{cm}^{-1}$. IVR is likely to occur on a subnanosecond timescale (Felker \& Zewail 1985), whereas the time available to absorb the photons is about $5 \mu$ s (i.e., the macropulse length). Competing decay mechanisms such as collisional relaxation (at the background pressure of about $10^{-6}$ torr) and infrared radiative decay typically take place on a millisecond timescale or even longer. Provided absorption strength and laser fluence are such to facilitate a sufficient photon absorption rate, multiple photons can be absorbed by a single molecule in which the absorbing mode has only its thermal excited-state population. Thus it is possible to avoid the shift away from resonance due to anharmonicities. During and after the macropulse, the ion 


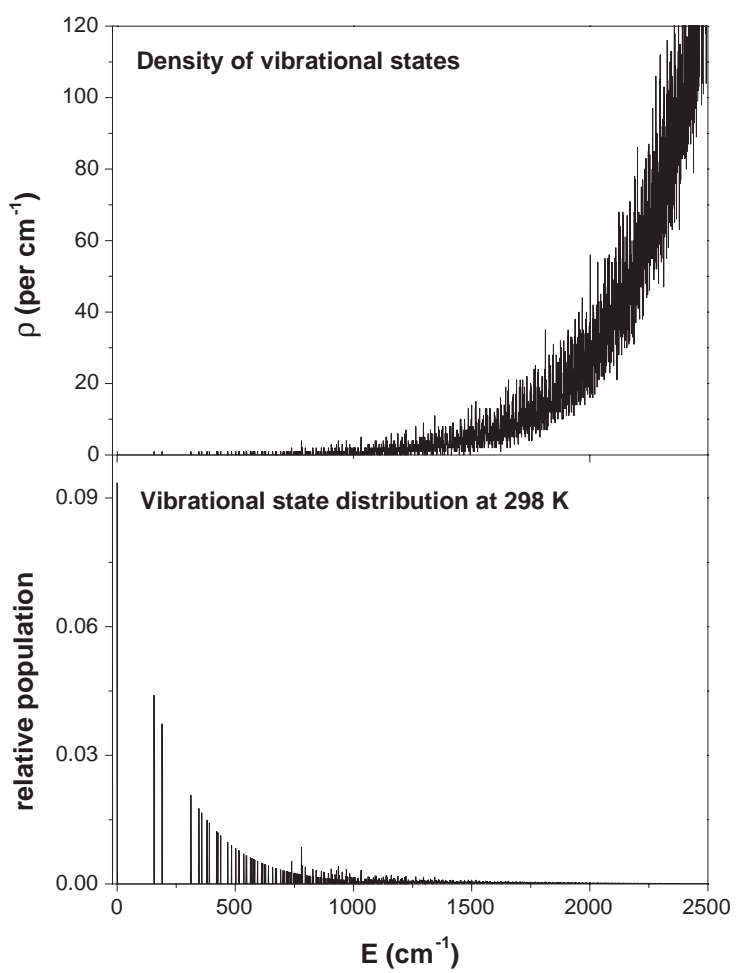

FIG. 4.-Calculated vibrational state density $\rho(E)$ for the naphthalene cation (top). Convolution with the Boltzmann factor and normalization gives the relative populations in the various vibrational levels at room temperature (bottom). Multiplying with the mode energy and integrating over all modes gives an average internal energy per molecule of $1006 \mathrm{~cm}^{-1}$.

can release its internal energy by infrared radiation, collisional quenching, or dissociation. Decay times for the former two mechanisms are typically in the millisecond to second range, whereas the dissociation rate for the naphthalene cation is calculated to be $10^{3} \mathrm{~s}^{-1}$ at $7 \mathrm{eV}$ internal energy and $10^{5} \mathrm{~s}^{-1}$ at $8 \mathrm{eV}$ (Gotkis et al. 1993).

Note that the bath of vibrational states not only aids in the sequential photon absorption process, but also yields a uniform fragmentation efficiency as it results in complete scrambling of the ion's memory of the initially excited vibrational mode. Consequently, fragmentation efficiency of the ion depends only on its total internal energy regardless of the vibrational mode through which the energy is pumped into the ion. For the larger PAH cations, the vibrational state densities are likely to be even higher, resulting in even shorter IVR lifetimes.

Recording the spectrum at different laser fluences reveals (only) slightly nonlinear line intensities, which may come as a surprise in view of the fact that more than 50 photons need to be absorbed to reach the dissociation threshold. As a possible explanation, it may be argued that the absorption of the first photon is the rate-limiting step in the process because the vibrational state density in the ion is still relatively low at this point. Such a single bottleneck would give rise to a linear dependence of the signal on laser power. As a first-order correction, all spectra were scaled linearly with laser power to compensate for variations in FELIX intensity. Despite the nonlinearities, relative spectral intensities as shown in Figure 2 compare fairly well with those in the linear absorption spectrum (Piest et al. 1999a).

\section{CONCLUSIONS}

Applying the intense radiation of a free electron laser, it has been possible to fragment PAH cations confined in a quadrupole ion trap. Fragmentation occurs whenever the laser radiation is in resonance with an allowed infrared transition in the ion. Thus, mass selective analysis of the contents of the trap as a function of wavelength allows one to record the infrared spectrum of the PAH ion in the gas phase. Care must be taken when interpreting the spectra, as line positions may be shifted slightly with respect to the ro-vibrationally cold ion, and, in addition, intensities may be affected by nonlinearities. However, elimination of unknown effects due to matrix isolation or van der Waals complexation may be regarded as an important asset. Compared with the messenger technique with which the naphthalene spectrum of Piest et al. (1999a) was recorded, the technique presented here is far simpler in various aspects; no molecular beam apparatus is required, nonresonant $\mathrm{ArF}$ ionization is applied instead of complex resonance enhanced multiphoton ionization (REMPI) schemes, and timing of the experiment is less critical.

The spectra obtained here compare favorably with available matrix isolation data though a redshift of typically $1 \%-2 \%$ is observed, most likely because of the difference in sample temperature (Joblin et al. 1995). Note, however, that the vibrational temperature prevailing in our experiments $(300 \mathrm{~K})$ matches that of the PAH cations giving rise to the UIR bands (300-1000 K; Le Coupanec et al. 1998; Joblin et al. 1995) relatively well, and thus spectral line shapes and shifts (due to anharmonic couplings) may be comparable. More specifically, one could consider the spectra shown here as absorption spectra averaged over cations with internal energies ranging from about $1000 \mathrm{~cm}^{-1}$ to several $\mathrm{eV}$, whereas the UIR emission spectra may be considered as averaged over cations cooling down over roughly the same energy range.

Combination of this method with more sophisticated mass-spectrometric techniques such as ion cyclotron resonance or high(er) resolution TOF detection may greatly improve the performance since, for example, the main $\mathrm{H}_{2}$ loss fragmentation channel may be resolved.

We gratefully acknowledge the expert technical assistance by the FELIX staff. We thank B. Sartakov for the many discussions on multiphoton absorption phenomena and A. G. G. M. Tielens for valuable comments on the manuscript. This work is part of the research program of FOM, which is financially supported by the Nederlandse Organisatie voor Wetenschappelijk Onderzoek (NWO).

\section{REFERENCES}

Allamandola, L. J., Hudgins, D. M., \& Sandford, S. A. 1999, ApJ, 511, L115 Allamandola, L. J., Tielens, A. G. G. M., \& Barker, J. R. 1985, ApJ, 290, L25

. 1989, ApJS, 71, 733

Boissel, P. 1995, in The Diffuse Interstellar Bands, ed. A. G. G. M. Tielens

\& T. P. Snow (Dordrecht: Kluwer), 221

Brenner, J. D., \& Barker, J. R. 1992, ApJ, 388, L39
Cook, D. J., Schlemmer, S., Balucani, N., Wagner, D. R., Steiner, B., \& Saykally, R. J. 1996, Nature, 380, 227

Ekern, S. P., Marshall, A. G., Szczepanski, J., \& Vala, M. 1998, J. Phys. Chem., 102, 3498

Felker, P. M., \& Zewail, A. H. 1985, J. Chem. Phys., 82, 2975

Fujii, A., Fujimaki, E., Ebata, T., \& Mikami, N. 2000, J. Chem. Phys., 112, 6275 
Gotkis, Y., Oleinikova, M., Naor, M., \& Lifshitz, C. 1993, J. Phys. Chem., 97,12282

Hudgins, D. M., \& Allamandola, L. J. 1995a, J. Phys. Chem., 99, 3033 .1995b, J. Phys. Chem., 99, 8978

. 1999, ApJ, 516, L41

Hudgins, D. M., Sandford, S. A., \& Allamandola, L. J. 1994, J. Phys. Chem., 98, 4243

Joblin, C., Boissel, P., Léger, A., d'Hendecourt, L., \& Défourneau D. 1995, A\&A, 299, 835

Joblin, C., d'Hendecourt, L., Léger, A., \& Défourneau, D. 1994, A\&A, 281, 923

Joblin, C., Tielens, A. G. G. M., Geballe, T. R., \& Wooden, D. H. 1996, ApJ, 460, L119

Jochims, H. W., Baumgärtel, H., \& Leach, S. 1999, ApJ, 512, 500

Langhoff, S. R. 1996, J. Phys. Chem., 100, 2819

Le Coupanec, P., Rouan, D., \& Léger, A. 1998, A\&A, 338, 217

Leger, A., \& Puget, J. L. 1984, A\&A, 137, L5

Oepts, D., Van der Meer, A. F. G., \& Van Amersfoort, P. W. 1995, Infrared Phys., 36, 297

Okumura, M., Yeh, L. I., Myers, J. D., \& Lee, Y. T. 1990, J. Phys. Chem., 94,3416

Paul, W. 1990, Rev. Mod. Phys., 62, 531

Pauzat, F., Talbi, D., Miller, M. D., DeFrees, D. J., \& Ellinger, Y. 1992, J. Phys. Chem., 96, 7882
Piest, J. A., Oomens, J., Bakker, J., von Helden, G., \& Meijer, G. 2000, Spectrochim. Acta, submitted

Piest, J. A., von Helden, G., \& Meijer, G. 1999a, ApJ, 520, L75 1999b, J. Chem. Phys., 110, 2010

Putter, M., von Helden, G., \& Meijer, G. 1996, Chem. Phys. Lett., 258, 118

Rouan, D., Léger, A., Omont, A., \& Giard, M. 1992, A\&A, 253, 498

Schlemmer, S., Cook, D. J., Harrison, J. A., Wurfel, B., Chapman, W., \& Saykally, R. J. 1994, Science, 265, 1686

Sloan, G. C., Hayward, T. L., Allamandola, L. J., Bregman, J. D., DeVito, B., \& Hudgins, D. M. 1999, ApJ, 513, L65

Stein, S. E., \& Rabinovitch, B. S. 1973, J. Chem. Phys., 58, 2438

Szczepanski, J., Roser, D., Personette, W., Eyring, M., Pellow, R., \& Vala, M. 1992, J. Phys. Chem., 96, 7876

Szczepanski, J., Vala, M., Talbi, D., Parisel, O., \& Ellinger, Y. 1993, J. Chem. Phys., 98, 4494

Todd, J. F. J., \& Penman, A. D. 1991, Int. J. Mass Spectrom. Ion Processes, 106,1

Vala, M., Szczepanski, J., Pauzat, F., Parisel, O., Talbi, D., \& Ellinger, Y. 1994, J. Phys. Chem., 98, 9187

von Helden, G., Holleman, I., Knippels, G. M. H., Van der Meer, A. F. G., \& Meijer, G. 1997, Phys. Rev. Lett., 79, 5234

von Helden, G., Holleman, I., Meijer, G., \& Sartakov, B. 1999, Opt. Expr., 4,46 